\title{
DETERMINATION OF THE WATER CONTENT OF SNOW FROM THE STUDY OF ELECTROMAGNETIC WAVE PROPAGATION IN THE SNOW GOVER
}

\author{
By J. Tobarias, P. Saguet and J. Chilo \\ (Laboratoire d'Électromagnétisme de l'École Nationale Supérieure d'Électronique et de \\ Radioélectricité de Grenoble, 23, rue des Martyrs, 3803i Grenoble Cedex, France)
}

\begin{abstract}
We propose a method for measuring in situ and continuously, the water content of a sample of snow in the snow cover. This method is based on the measurement of the attenuation of an electromagnetic wave propagating in a sample of snow situated between two antennae, an emitter and a receiver. The working frequency is $9.4 \mathrm{GHz}$.

Résumé. Détermination de la teneur en eau de la neige à partir de l'étude de la propagation d'une onde électromagnétique dans le manteau neigeux. Nous proposons une méthode permettant de mesurer in situ et d'une façon continue, la teneur en eau d'un échantillon de neige dans un manteau neigeux. Cette méthode est basée sur la mesure de l'atténuation d'une onde électromagnétique se propageant dans un échantillon de neige situé entre deux antennes émettrice et réceptrice. La fréquence de travail est de $9,4 \mathrm{GHz}$.

Zusammenfassung. Bestimmung des Wassergehalts von Schnee durch Untersuchung der Ausbreitung elektromagnetischer Wellen in der. Schneedecke. Eine Methode zur kontinuierlichen in situ-Messung des Wassergehalts einer Schneeprobe in der Schneedecke wird vorgeschlagen. Die Methode beruht auf der Messung der Dämpfung einer elektromagnetischen Welle, die sich in einer Schneeprobe zwischen Sender- und Empfängerantenne ausbreitet. Die Arbeitsfrequenz ist $9,4 \mathrm{GHz}$.
\end{abstract}

\section{INTRODUCTION}

The determination of the change with time of the parameter water content $W$ is a complementary measurement to those of radiation balance and thermal exchange at the surface of the snow cover. It also allows percolation into the snow mantle during the melting period to be followed. In addition, in the study of wet-snow avalanches in spring, knowledge of $W$ would enable prediction of critical conditions for avalanche release (Colbeck, I973).

Different methods have been proposed for measuring the water content of snow (Yosida, 1967): by calorimetry, by centrifugation, or by measurement of the permittivity of snow at $20 \mathrm{MHz}$ (Ambach and Denoth, 1972). The first two methods can only be carried out in the laboratory, and moreover the centrifugation technique is not very accurate for low watercontent values. Permittivity measurements can be made in situ but, as with both the other methods, only instantaneous values can be obtained.

We propose a method which enables continuous monitoring of the water content of a sample of snow in a natural snow mantle. It is based on the measurement of the attenuation of an electromagnetic wave propagating in a sample of snow situated between two antennae, an emitter and a receiver.

The working frequency is chosen to be in the $\mathrm{S}$ and $\mathrm{X}$ bands (in the range of the dielectric relaxation of water). The dielectric properties of water and ice are very different at these frequencies and the influence of the two components on the permittivity of the wet snow can readily be separated.

The attenuation measurement allows the water content $W$ to be determined if the dielectric properties are known as a function of this parameter. We have measured these in the laboratory (Tobarias, unpublished) and the results are shown in Figure I for granular snow of density $0.5 \mathrm{Mg} \mathrm{m}^{3}$ at a frequency of $9.4 \mathrm{GHz}$.

The direction of wave propagation can be chosen to be perpendicular or parallel to the snow surface. Only the latter configuration allows measurements of $W$ to be made independently of the thickness of the wet-snow layer (as we will demonstrate) and thus we consider parallel propagation. 


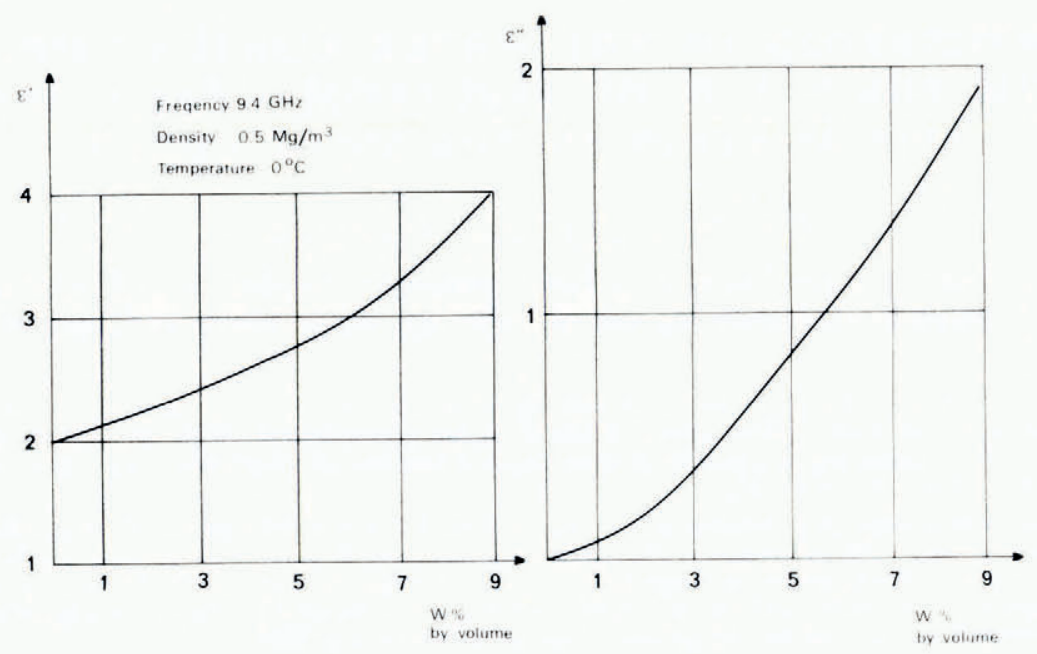

Fig. I. Complex relative permittivity of wet snow.

\section{The theory of PARALlel Propagation}

\section{Idealized snow-cover model}

We consider the snow cover to be of constant depth $h$ lying on level ground with the wet snow constituting a layer of uniform thickness $d$ at the surface of the mantle. We assume $d \ll h$ (Fig. 2).

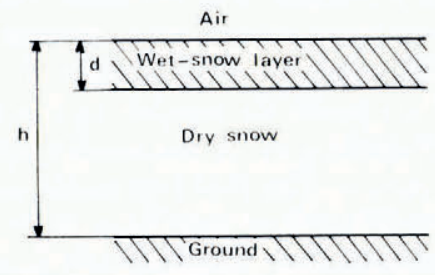

Fig. 2. Idealized model of snow cover.

\section{Solution of Maxwell's equations}

The three dielectric media are separated by plane interfaces perpendicular to the $\mathrm{O} x$. axis and infinite in the $\mathrm{O} y$ and $\mathrm{O} z$ directions. Media (1) and (3) are semi-infinite along $x>0$ and $x<0$ respectively (for $d \ll h$, Fig. 3). Propagation is in the $\mathrm{O} z$ direction. For each medium $j,(j=\mathrm{I}, 2,3)$ and for each axis $p,(p=x, y, z)$ the field components $E_{j p}$. have, in the general case, different complex propagation constants $k_{j p}$.

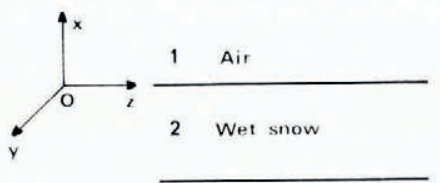

3 Dry snow

Fig. 3. Coordinate system and labelling of media. 
The wave equation can be written:

$$
\Delta E_{j p}-\mu_{0} \epsilon_{0} \epsilon_{j} \frac{\partial^{2} E_{j p}}{\partial t^{2}}=0
$$

where $\epsilon_{j}$ is the complex relative permittivity of medium $j$. We look for solutions of the form:

$$
E_{j p}(x, y, z, t)=E_{\text {ojp }}(x) \exp \mathrm{i}\left(\omega t-k_{j p} z\right),
$$

such that $E_{j p} \rightarrow$ o as $x \rightarrow \pm \infty$.

By developing Maxwell's equations and writing field continuity at the interfaces, we have shown (Tobarias, unpublished) that two possible sets emerge, one $E_{x}, E_{z}, H_{z}$ of propagation constant $k_{\text {TM }}$ describing a "transverse magnetic" wave and the other $E_{y}, H_{x}, H_{z}$ of propagation constant $k_{\text {TE }}$ describing a "transverse electric" wave.

3. "Transverse electric" wave

This wave is described by the equations:

$$
\left.\begin{array}{l}
E_{1 y}=A \exp (-\alpha x) \exp \mathrm{i}\left(\omega t-k_{\mathrm{TE}} z\right), \\
E_{2 y}=[B \exp \mathrm{i} \beta x+C \exp (-\mathrm{i} \beta x)] \exp \mathrm{i}\left(\omega t-k_{\mathrm{TE}} z\right), \\
E_{3 y}=D \exp \gamma x \exp \mathrm{i}\left(\omega t-k_{\mathrm{TE}} z\right),
\end{array}\right\}
$$

where

$$
\left.\begin{array}{llll}
\alpha=\left(k_{\mathrm{TE}^{2}}-k_{0}^{2} \epsilon_{\mathrm{I}}\right)^{\frac{1}{2}} & \text { and } & \operatorname{Re}(\alpha)>0, & \text { (a) } \\
\beta=\left(k_{0}^{2} \epsilon_{2}-k_{\mathrm{TE}}^{2}\right)^{\frac{1}{2}} & \text { and } & \operatorname{Re}(\beta)>0, & \text { (b) } \\
\gamma=\left(k_{\mathrm{TE}^{2}}-k_{0}^{2} \epsilon_{3}\right)^{\frac{1}{2}} & \text { and } & \operatorname{Re}(\gamma)>0 . & \text { (c) }
\end{array}\right\}
$$

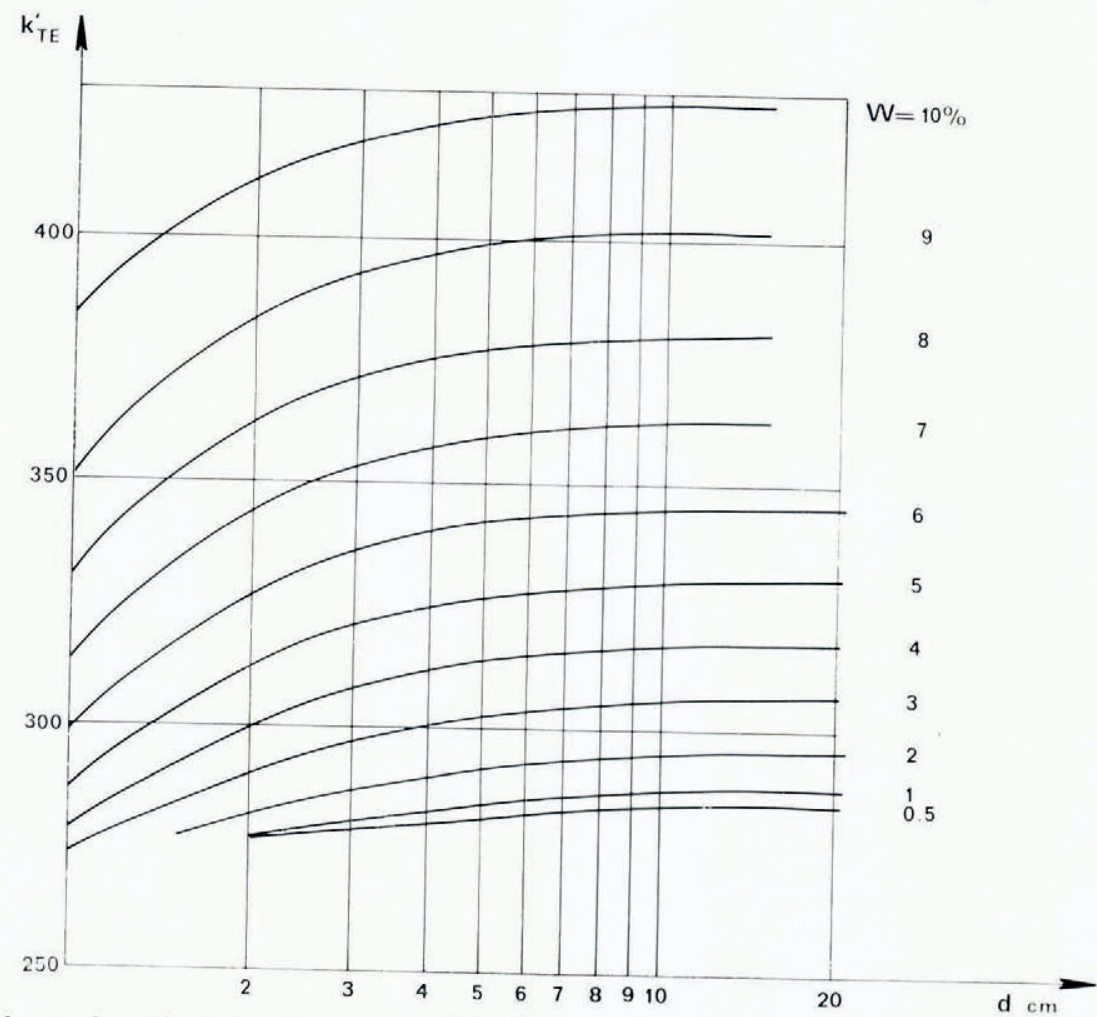
Fig. 4. Real part of complex propagation constant of the TE wave plotted as a function of thickness of wet snow for various
values of the water content. 
Field continuity at the interfaces leads to the dispersion equation:

$$
\beta d=\arctan \frac{\alpha}{\beta}+\arctan \frac{\gamma}{\beta}+n \pi \quad \text { for } n=0,1,2, \ldots .
$$

\section{I. Fundamental mode $\mathrm{TE}_{0}(n=0)$}

In Figures 4 and 5 we have plotted the complex propagation constant $k_{\mathrm{TE}}=k_{\mathrm{TE}}{ }^{\prime}-\mathrm{i} k_{\mathrm{TE}}{ }^{\prime \prime}$ as a function of the thickness $d$ of a wet snow layer at $9 \cdot 4 \mathrm{GHz}$.

The following values of permittivity have been used in the calculations:

$$
\begin{aligned}
\epsilon_{\mathrm{I}} & =\epsilon_{\mathrm{I}}{ }^{\prime}=\mathrm{I}, \\
\epsilon_{3} & =\epsilon_{3}{ }^{\prime}-\mathrm{i} \epsilon_{3}{ }^{\prime \prime}=2-2 \times 10^{-3} \mathrm{i}, \\
\epsilon_{2}(W) & =\epsilon_{2}{ }^{\prime}-\mathrm{i} \epsilon_{2}{ }^{\prime \prime}
\end{aligned}
$$

found from curves in Figure $I$.

\subsection{Harmonic modes $\mathrm{TE}_{n}(n \neq 0)$}

The study of these modes shows that they can be easily ignored by correctly exciting the wave propagating in the wet-snow layer.

\section{4. "Transverse magnetic" wave}

In this case we obtain the following dispersion equation:

$$
\beta^{\prime} d=\arctan \frac{\gamma^{\prime} \epsilon_{2}}{\beta^{\prime} \epsilon_{3}}+\arctan \frac{\alpha^{\prime} \epsilon_{2}}{\beta^{\prime} \epsilon_{\mathrm{I}}}+m \pi \quad \text { for } m=0, \mathrm{I}, 2, \ldots,
$$

where

$$
\left.\begin{array}{llll}
\alpha^{\prime}=\left(k_{\mathrm{TM}}^{2}-k_{\mathrm{o}}^{2} \epsilon_{\mathrm{I}}\right)^{\frac{1}{2}}, & \text { and } & \operatorname{Re}\left(\alpha^{\prime}\right)>0, & \text { (a) } \\
\beta^{\prime}=\left(k_{0}^{2} \epsilon_{2}-k_{\mathrm{TM}}\right)^{\frac{1}{2}}, & \text { and } & \operatorname{Re}\left(\beta^{\prime}\right)>0, & \text { (b) } \\
\gamma^{\prime}=\left(k_{\mathrm{TM}}^{2}-k_{0}^{2} \epsilon^{2}\right)^{\frac{1}{2}}, & \text { and } & \operatorname{Re}\left(\gamma^{\prime}\right)>0 . & \text { (c) }
\end{array}\right\}
$$

We have plotted the propagation constant $k_{\mathrm{TM}}=k_{\mathrm{TM}}{ }^{\prime}-\mathrm{i} k_{\mathrm{TM}}{ }^{\prime \prime}$ for the fundamental mode under the same conditions as for the $\mathrm{TE}_{0}$ mode (Figs 6 and 7 ).

\section{Conclusion}

The propagation of the $\mathrm{TE}_{0}$ and $\mathrm{TM}_{0}$ modes is only possible for values of thickness $d$ greater than a minimum value $d_{\mathrm{m}}$ which varies with parameter $W$. If we take $d<d_{\mathrm{m}}$, the dispersion equation no longer gives solutions which satisfy the Conditions (4) (particularly 4c). For low values of water content we have for the $\mathrm{TE}_{0}$ mode

$$
d_{\mathrm{m}}=\frac{\mathrm{I}}{k_{0}\left(\epsilon_{2}{ }^{\prime}-\epsilon_{3}{ }^{\prime}\right)^{\frac{1}{2}}} \arctan \left(\frac{\epsilon_{3}{ }^{\prime}-\epsilon_{1}{ }^{\prime}}{\epsilon_{2}{ }^{\prime}-\epsilon_{3}{ }^{\prime}}\right)^{\frac{1}{2}} .
$$

while for the $\mathrm{TM}_{0}$ mode

$$
d_{\mathrm{m}}=\frac{\mathrm{I}}{k_{0}\left(\epsilon_{2}{ }^{\prime}-\epsilon_{3}\right)^{\frac{1}{2}}} \arctan \left(\frac{\epsilon_{3}{ }^{\prime}-\epsilon_{\mathrm{I}}{ }^{\prime}}{\epsilon_{2}{ }^{\prime}-\epsilon_{3}{ }^{\prime}}\right)^{\frac{1}{2}} \frac{\epsilon_{2}{ }^{\prime}}{\epsilon_{\mathrm{I}}{ }^{\prime}} .
$$

Figures 5 and 6 show that the attenuation constants $k_{\mathrm{TE}}{ }^{\prime \prime}$ and $k_{\mathrm{TM}}{ }^{\prime \prime}$ tend to a single limiting value $k_{W}{ }^{\prime \prime}$ for thickness $d$ greater than the wavelength. This limiting value $k_{W}{ }^{\prime \prime}$ is a function of $W$ only.

The dispersion equations (5) and (6) give

$$
\lim k_{\mathrm{TE}^{2}}=\lim k_{\mathrm{TM}^{2}}=k_{0}^{2} \epsilon_{2}=k_{W^{2}} .
$$



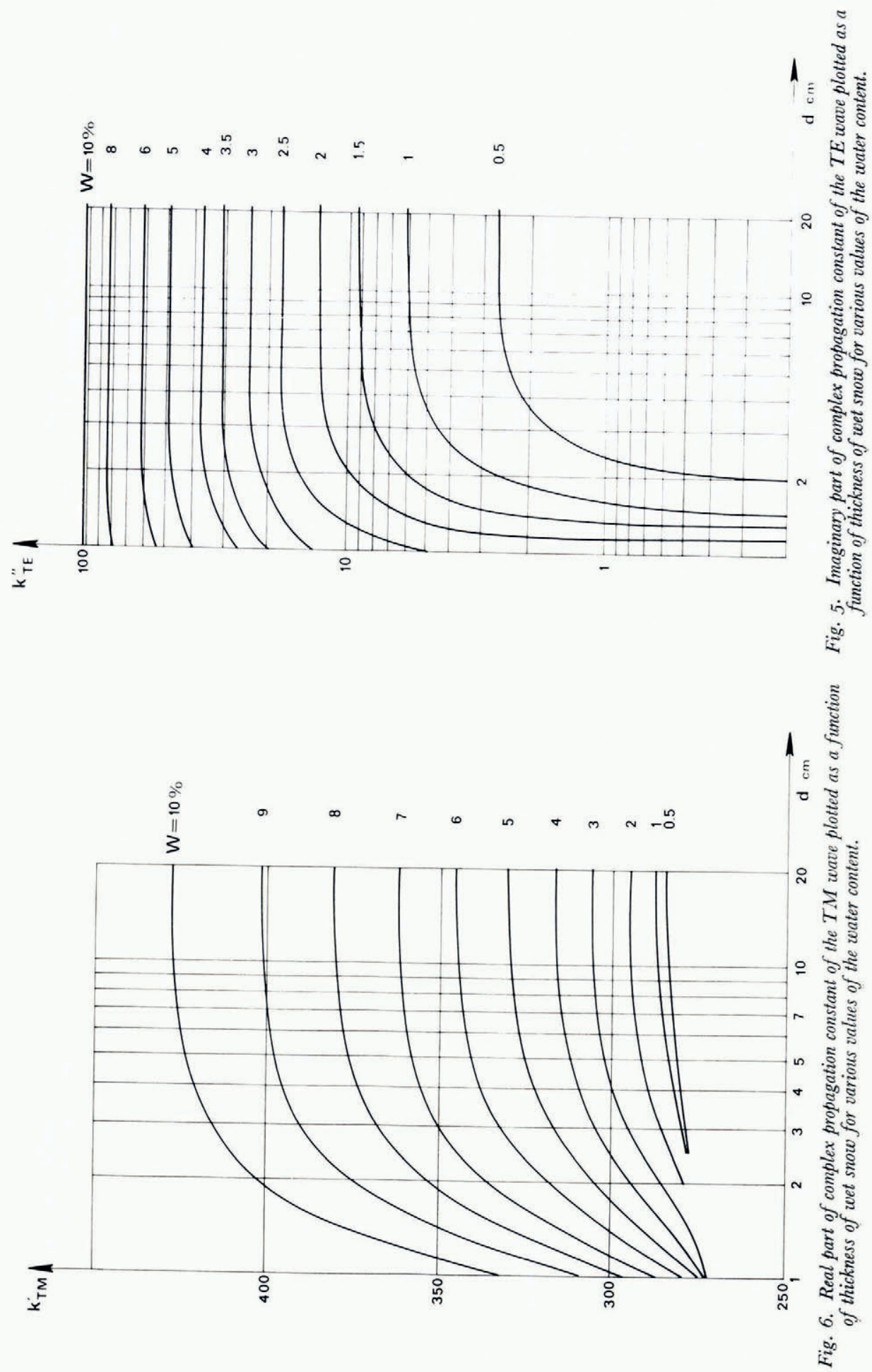

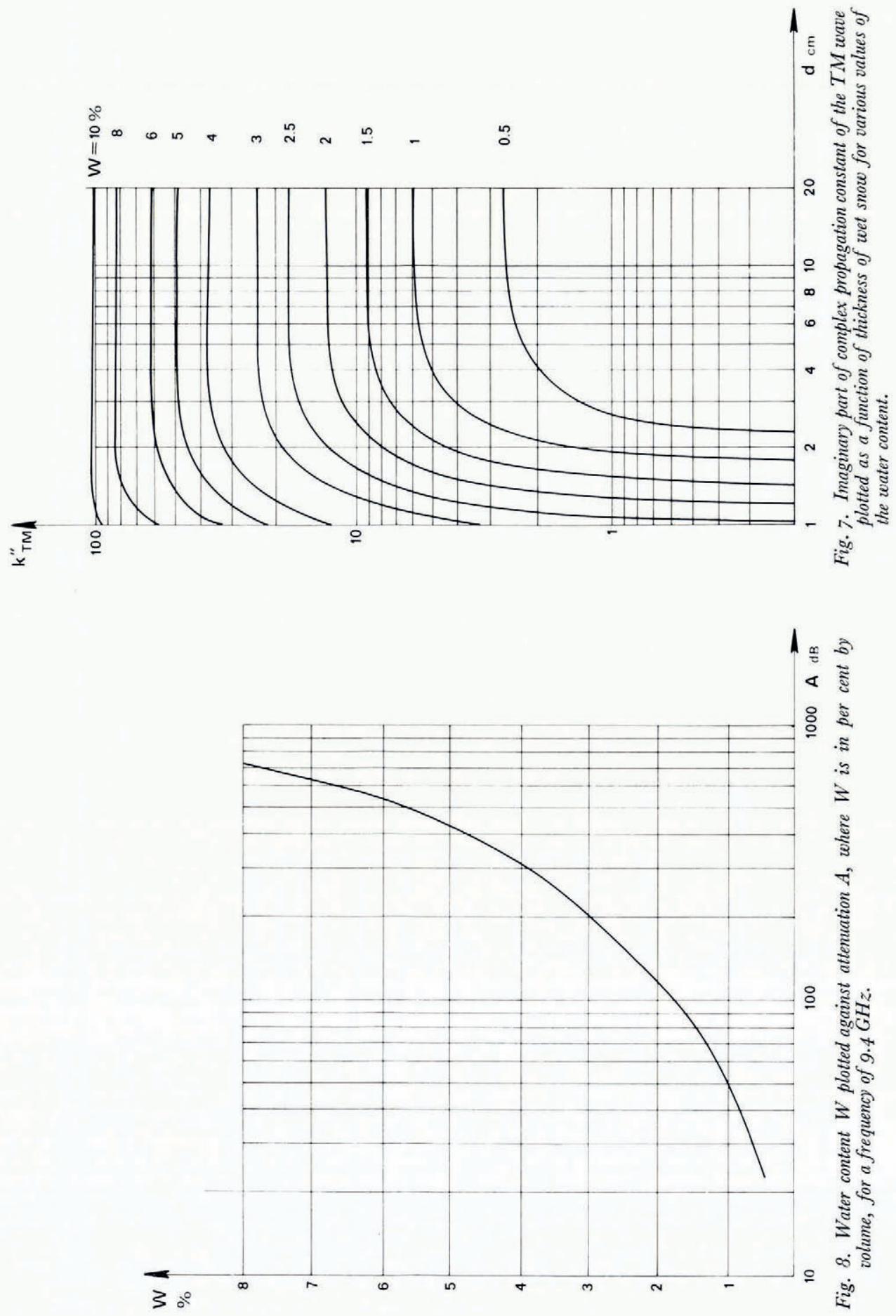
The attenuation of an electromagnetic wave propagating in a layer of wet snow is a relatively complicated function of $W$. If we assume a sufficient thickness $d$ and a large enough distance $l$ between the antennae (to avoid standing waves). The attenuation $A$ will be proportional to $\exp \left(-k_{W}{ }^{\prime \prime} l\right)$. That is, if $A$ is measured in decibels,

$$
A=8.686 k_{W}^{\prime \prime} l \text {. }
$$

The measurement of $A$ for a given length $l$ allows $k_{W}$ " to be found and hence the water content $W$ (Fig. 8).

The $\mathrm{TE}_{0}$ and $\mathrm{TM}_{0}$ modes are obtained by orienting the emitting horn antenna so that the radiated electric field has the appropriate polarization: along $\mathrm{O} x$ for the $\mathrm{TM}_{0}$ mode, and along $\mathrm{O} y$ for the $\mathrm{TE}_{0}$ mode.

The measuring device used in the basic experiment includes two antennae, an emitter and a receiver, introduced in the snow cover near its surface (Tobarias, unpublished) so that the radiated energy is concentrated in the wet-snow layer of thickness $d$ (Fig. 9).

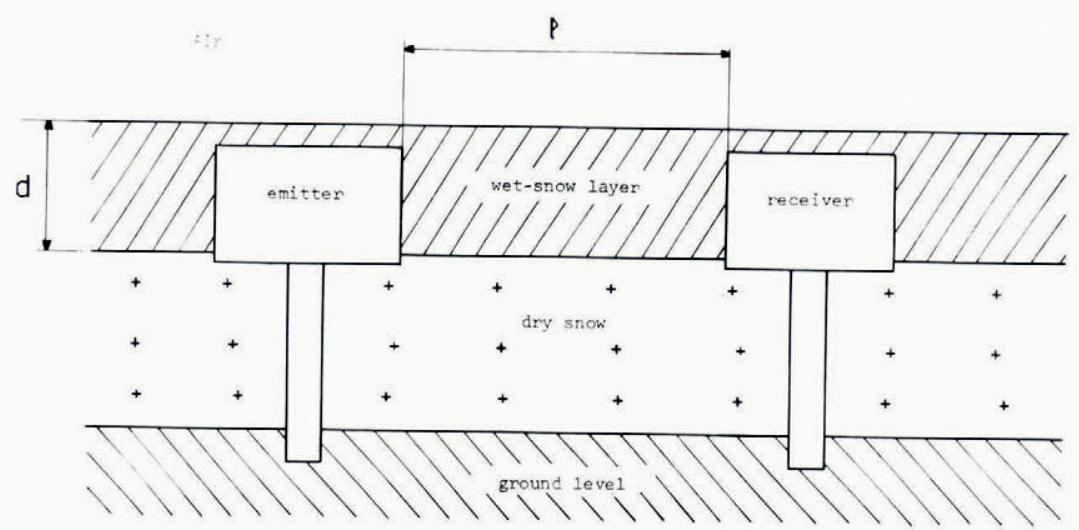

Fig. 9. Sketch of the basic experiment.

We suppose the thickness $d$ to be greater than the working wavelength, hence we can take the limiting value $k_{W}$ " for the imaginary part of the propagation constants $k_{\mathrm{TE}}$ and $k_{\mathrm{TM}}$.

The emitting antenna is a horn antenna with an electromagnetic lens which enables quasi-plane waves propagating in the $\mathrm{O} z$ direction (Fig. 3) to be obtained. The distance between the antennae $(l=\mathrm{I} .2 \mathrm{~m})$ is sufficient to eliminate any standing wave.

We measure the attenuation of an electromagnetic wave propagating in the snow sample situated between the two antennae. From the attenuation factor $A$, we can determine the value of $W$ (Fig. 8). cation.

The apparatus used and the results obtained will be described in a subsequent communi-

\section{Acknowledgement}

We gratefully acknowledge the assistance of $\mathrm{Mr} \mathrm{N}$. M. Harris in the preparation of this communication.

MS. received 25.7 uly 1977 and in revised form 7 March 1978 


\section{REFERENCES}

Ambach, W., and Denoth, A. 1972. Studies on the dielectric properties of snow. Zeitschrift für Gletscherkunde und Glazialgeologie, Bd. 8, Ht. $\mathrm{I}-2$, p. $113^{-23}$.

Colbeck, S. C. 1973. Theory of metamorphism of wet snow. U.S. Cold Regions Research and Engineering Laboratory. Research Report 3 13.

Tobarias, J. Unpublished. Propriétés diélectriques de la neige. Application à la mesure de la teneur en eau. [Dr 3 ème Cycle thesis, Institut National Polytechnique de Grenoble, 1977.]

Yosida, Z. [i.e. Yoshida, J.] 1967. Free water content of wet snow. (In Oura, H., ed. Physics of snow and ice: international conference on low temperature science. . . 1966. . . Proceedings, Vol. I, Pt. 2. [Sapporo], Institute of Low Temperature Science, Hokkaido University, p. 773-84.) 